\title{
Angiographic Analysis of Natural Anastomoses between the Posterior and Anterior Cerebral Arteries in Moyamoya Disease and Syndrome
}

\author{
(D) S. Bonasia, (D) G. Ciccio, (D) S. Smajda, (D) A.G. Weil, (D). Chaalala, (DR. Blanc, (D) M. Reinert, (D). Piotin, (D). Bojanowski, and
}

(iD) $\mathrm{T}$. Robert

\begin{abstract}
BACKGROUND AND PURPOSE: Moyamoya disease is a chronic neurovascular steno-occlusive disease of the internal carotid artery and its main branches, associated with the development of compensatory vascular collaterals. Literature is lacking about the precise description of these compensatory vascular systems. Usually, the posterior circulation is less affected, and its vascular flow could compensate the hypoperfusion of the ICA territories. The aim of this study was to describe these natural connections between the posterior cerebral artery and the anterior cerebral artery necessary to compensate the lack of perfusion of the anterior cerebral artery territories in the Moyamoya population.
\end{abstract}

MATERIALS AND METHODS: All patients treated for Moyamoya disease from 2004 to 2018 in 4 neurosurgical centers with available cerebral digital subtraction angiography were included. Forty patients ( 80 hemispheres) with the diagnosis of Moyamoya disease were evaluated. The presence of anastomoses between the posterior cerebral artery and the anterior cerebral artery was found in 31 hemispheres (38.7\%).

RESULTS: Among these 31 hemispheres presenting with posterior cerebral artery-anterior cerebral artery anastomoses, the most frequently encountered collaterals were branches from the posterior callosal artery (20\%) and the posterior choroidal arteries (20\%). Another possible connection found was pio-pial anastomosis between cortical branches of the posterior cerebral artery and the anterior cerebral artery (15\%). We also proposed a 4-grade classification based on the competence of these anastomoses to supply retrogradely the territories of the anterior cerebral artery.

CONCLUSIONS: We found 3 different types of anastomoses between the anterior and posterior circulations, with different abilities to compensate the anterior circulation. Their development depends on the perfusion needs of the territories of the anterior cerebral artery and can provide the retrograde refilling of the anterior cerebral artery branches.

ABBREVIATIONS: $\mathrm{ACA}=$ anterior cerebral artery; $\mathrm{PCA}=$ posterior cerebral artery

M oyamoya disease is a neurovascular pathology characterized by the progressive stenosis of the supraclinoidal portion of the ICA and its 2 main branches: the anterior cerebral artery (ACA) and MCA in their proximal segments. ${ }^{1-3}$ During the evolution of the pathology, the development of natural collaterals allows maintaining a minimal perfusion to the cerebral parenchyma and, in particular, the ACA territories. ${ }^{2,4,5}$ The knowledge

Received August 9, 2019; accepted after revision September 10.

From the Department of Neurosurgery (S.B., M.R., T.R.), Neurocenter of the Southern Switzerland, Lugano, Switzerland; Department of Interventional

Neuroradiology (G.C., S.S., R.B., M.P.), Rothschild Foundation Hospital, Paris, France Department of Pediatric Neurosurgery (A.G.W.), Sainte-Justine Hospital, Montreal, Quebec, Canada; Department of Neurosurgery (C.C., M.B.), Centre Hospitalier de

l'Université de Montréal, Montreal, Quebec, Canada; and Faculty of Medicine (M.R., T.R.), University of the Southern Switzerland, Lugano, Switzerland.

Please address correspondence to Sara Bonasia, MD, Regional Hospital of Lugano, Department of Neurosurgery, Neurocenter of the Southern Switzerland, Via Tesserete 46, CH-6903 Lugano, Switzerland; e-mail: sara.bonasia@gmail.com

http://dx.doi.org/10.3174/ajnr.A6291 of the collateral circles and the specific connections that the patient develops during the natural history of this disease is still not well-known, and just a few studies in the literature are dedicated to their analysis. ${ }^{6-9}$ In a recent study, ${ }^{8}$ we described the role of collaterals from the ophthalmic artery in supplying the ACA territory. However, the other collateral circles are still not known in detail. Due to this important absence in the literature, we proposed to analyze the other collateral circles in Moyamoya disease. This study aimed to describe angiographically the anastomotic circulation between the posterior cerebral artery (PCA) and the ACA, which maintains correct perfusion of the ACA territory.

\section{MATERIALS AND METHODS \\ Patient Selection}

On the basis of a prospective data base from 2004 to 2018, fiftysix patients with the diagnosis of Moyamoya disease or syndrome 
have been evaluated in our institutions (Sainte Justine Hospital, Montreal; Notre-Dame Hospital, Montreal; Rothschild Foundation Hospital, Paris; Regional Hospital, Lugano). The patients had Moyamoya syndrome with characteristic Moyamoya vasculopathy and the following associated conditions: unilateral vascular changes, sickle cell disease, neurofibromatosis type 1 , and cranial therapeutic irradiation. All patients who underwent at least 1 DSA were included in our study (40 patients). The other 16 patients with Moyamoya disease who were only evaluated by MR imaging were excluded. Therefore, a retrospective angiographic study of anastomoses between the posterior and anterior circulations was possible in 80 hemispheres (40 patients). We looked for the presence of an etiology, the duration of symptoms, the type of clinical sign (ischemic or hemorrhagic), and the clinical outcome.

\section{Analysis of the Posterior Circulation Collateral Anatomy}

A 6-vessel DSA with the patient under local anesthesia with 3D reconstruction was performed on all patients of this study. All DSA interpretation was performed by the same 2 senior neuroradiologists, and in case of disagreement, a third neuroradiologist was consulted. The Suzuki grading system ${ }^{10}$ was applied to each cerebral hemisphere. As suggested by Baltsavias et al, ${ }^{6,7}$ different arterial collaterals were categorized into leptomeningeal (pio-pial), duro-pial, and periventricular collaterals. For each hemisphere evaluated, careful attention was paid to the following: the presence of an anastomotic connection between the posterior cerebral artery and the anterior cerebral artery, the number of vessels involved, their exact anatomy, and the competence of the anastomosis to supply by retrograde flow the ACA territories.

\section{Treatment and Clinical Evolution}

Modalities of medical and surgical treatments were recorded. Follow-up started at the time of the first clinical sign and ended with the last clinical visit. A neurologic examination with evaluation of the mRS was systematically performed for each visit. Most patients underwent only 1 DSA for the diagnostic radiologic assessment of the pathology and then were followed with cerebral MR imaging.

\section{Data Availability}

The data cannot be shared for ethical reasons because patients involved were mostly younger than 18 years of age and gave no consent for data sharing.

\section{Statistical Analysis}

A complex statistical analysis is not required for this kind of study. Percentage calculations were performed by Sara Bonasia, MD (Neurocenter of Southern Switzerland), and the detailed data are summarized in Tables 1-3.

\section{RESULTS}

\section{Clinical Presentation}

Between 2004 and 2018, forty patients fulfilled the inclusion criteria for this study. Patient baseline data and clinical signs are summarized in Tables $1-3$. The mean age was 40.9 years
Table 1: Demographic and clinical data

\begin{tabular}{lc}
\hline \multicolumn{1}{c}{ Variable } & Patients $(\boldsymbol{n}=\mathbf{4 0})$ \\
\hline Female & $29(72.5 \%)$ \\
Sex ratio, F/M & $2.6: 1$ \\
Median age (yr) & $40.9(1-64)$ \\
Clinical presentation & \\
Ischemic & $23(57.5 \%)$ \\
Hemorrhagic & $13(32.5 \%)$ \\
SAH & $9(22.5 \%)$ \\
Intraparenchymal & $4(10 \%)$ \\
Baseline mRS & \\
0 & $1(2.5 \%)$ \\
1 & $12(30 \%)$ \\
2 & $15(37.5 \%)$ \\
3 & $5(12.5 \%)$ \\
4 & $1(2.5 \%)$ \\
5 & $6(15 \%)$ \\
\hline
\end{tabular}

Table 2: Suzuki stage of the population

\begin{tabular}{lc}
\hline \multicolumn{1}{c}{ Suzuki Stage } & Hemispheres $(\boldsymbol{n}=\mathbf{8 0})$ \\
\hline 0 & $10(12.5 \%)$ \\
I & $10(12.5 \%)$ \\
II & $10(12.5 \%)$ \\
III & $14(17.5 \%)$ \\
IV & $16(20 \%)$ \\
V & $7(8.8 \%)$ \\
VI & $11(13.8 \%)$ \\
NA (Cervical ICA occlusion) & $2(2.5 \%)$ \\
\hline
\end{tabular}

Note:-NA indicates not applicable.

\section{Table 3: Results}

\begin{tabular}{lc}
\multicolumn{1}{c}{ Origin of the Collaterals } & $\begin{array}{c}\text { Hemispheres }(\boldsymbol{n}= \\
80)\end{array}$ \\
\hline $\begin{array}{l}\text { Collaterals from posterior callosal artery } \\
\text { (type I) }\end{array}$ & $16(20 \%)$ \\
$\begin{array}{l}\text { Collaterals from posterior choroidal arteries } \\
\text { (type II) }\end{array}$ & $16(20 \%)$ \\
Pio-pial connections (type III) & $12(15 \%)$ \\
Competence & \\
Type I & $7(8.7 \%)$ \\
Type II & $12(15 \%)$ \\
Type III & $9(11.3 \%)$ \\
Type IV & $5(6.3 \%)$ \\
Correlation between Suzuki score and & \\
posterior collaterals & \\
O & $1(1.3 \%)$ \\
I & $1(1.3 \%)$ \\
II & $4(5 \%)$ \\
III & $4(5 \%)$ \\
IV & $8(10 \%)$ \\
V & $4(5 \%)$ \\
VI & $8(10 \%)$ \\
Cervical occlusion of the ICA & $1(1.3 \%)$ \\
\hline
\end{tabular}

(range, 1-64 years) with a female preponderance (female/male ratio, 2.6:1). The most common clinical sign was an ischemic stroke in 23 patients $(57.5 \%)$. Other patients presented with hemorrhage (13 patients, 32.5\%). Among them, 4 patients had intraparenchymal bleeding; and 9 patients, a subarachnoid hemorrhage. The initial mRS score was 0 in 1 patient $(2.5 \%), 1$ in 12 patients (30\%), 2 in $15(37.5 \%), 3$ in $5(12.5 \%), 4$ in 1 $(2.5 \%)$, and 5 in $6(15 \%)$. 


\section{Angiographic Analysis of Moyamoya Disease}

We analyzed the presence of collaterals between the PCA and the ACA for each of the 80 cerebral hemispheres. The Suzuki stage was 0 in 10 hemispheres (12.5\%), I in 10 (12.5\%), II in 10 (12.5\%), III in 14 hemispheres (17.5\%), IV in $16(20 \%), \mathrm{V}$ in 7 $(8.75 \%)$, and VI in 11 hemispheres (13.75\%). Two patients $(2.5 \%)$ had a cervical occlusion of the ICA; thus, the Suzuki classification was not applicable. Among the hemispheres studied, the posterior communicating artery had a normal configuration (P1 segment larger than posterior communicating artery) in 56 cases (70\%) and a fetal configuration (posterior communicating artery larger than P1 segment) in 24 cases (30\%).

The observation of angiographies led us to identify different types of connections between the anterior and posterior circulations. We classified the different anastomoses observed into 3 types: In type I, the posterior pericallosal or splenial artery has anastomosis with the terminal branches of the anterior pericallosal artery; in type II, the medial posterior choroidal artery has posterior anastomosis with terminal branches of the anterior pericallosal artery; in type III, cortical branches of the PCA create pio-pial (leptomeningeal) connections to cortical ACA branches. Figure 1 shows some examples of the 3 types of PCA-ACA anastomoses.

The presence of collaterals from the posterior circulation was noted in 34 hemispheres, but 3 of them were excluded from our analysis because they had a connection between the PCA and the MCA and not with the ACA. Angiographic results of our study are summarized in Table 3. Among the 31 hemispheres (38.7\% of the total) included, we found collaterals to the ACA from the posterior pericallosal or splenial artery (type I) in 16 hemispheres (20\%), from the medial posterior choroidal artery (type II) in 16 hemispheres (20\%), and a pio-pial connection (type III) in 12 hemispheres (15\%). Figure 2 shows the Suzuki score and the presence of each type of PCA-ACA anastomosis in the different age groups of our population. Patients younger than 20 years of age and older than 50 years of age are the 2 groups in whom the presence of PCA-ACA anastomosis was the most frequent.

The presence of natural collaterals between branches of the ophthalmic artery and branches of the ACA was noted in 35 hemispheres (43.8\%). There is no numerical difference among the different groups. Twenty-one hemispheres presented with ophthalmic artery-ACA anastomosis without PCA-ACA anastomosis, 5 presented with ophthalmic artery-ACA concomitantly with a PCA-ACA type I anastomosis; 5 others, ophthalmic artery-ACA and PCA-ACA type II anastomosis; and 4 hemispheres presented with ophthalmic artery-ACA concomitantly with a PCA-ACA type III anastomosis.

We also analyzed the competence of each anastomosis in supplying the ACA vascular territories. According to the importance of retrograde supply of the ACA territories, we proposed a 4grade classification that is illustrated in Fig 3. Grade I corresponds to anastomosis allowing the visualization of the first part of the posterior pericallosal artery without seeing any cortical ACA branch (7 hemispheres, 8.7\%). The grade II is noted when the retrograde flow in the ACA is important enough to see 1 cortical branch (12 hemispheres, 15\%). Grade III corresponds to an anastomosis important enough to visualize $\geq 2$ cortical branches of the posterior pericallosal artery (9 hemispheres, 11.25\%). Grade IV consists of the complete retrograde refilling of the ACA (5 hemispheres, 6.25\%).

\section{Collaterals from the Posterior Pericallosal (Splenial) Artery}

The most frequently encountered anastomosis between the posterior cerebral artery and the anterior cerebral artery comes from a branch of the posterior pericallosal artery or splenial artery. This connection was found in $20 \%$ of cases ( 16 hemispheres), and one of these cases is shown in Fig $1 A$. The posterior pericallosal artery normally arises from the PCA or its branches and supplies the splenium of the corpus callosum. ${ }^{11}$ It can anastomose with the anterior pericallosal artery, a branch of the ACA, to fill retrogradely the latter artery.

\section{Collaterals from Posterior Choroidal Arteries}

The other frequent collaterals we found are those from the medial posterior choroidal artery. The choroidal arteries could be divided into a medial and lateral system (medial posterior choroidal artery and lateral posterior choroidal artery), with the former arising from the P1 segment or at the junction of the P1-P2 segment and the latter originating from the $\mathrm{P} 2$ segment. ${ }^{12}$ They anastomose with the branches of the anterior choroidal artery and the anterior cerebral artery to supply the choroidal plexus. ${ }^{11}$ In detail, the medial posterior choroidal artery divides into a lateral branch, which anastomoses into the lateral ventricle with branches of the anterior choroidal artery or with the lateral posterior choroidal artery and into a medial branch, which anastomoses on the roof of the third ventricle with the contralateral medial posterior choroidal artery. The lateral posterior choroidal artery enters the lateral ventricle in the temporal horn or in the atrium, and here it anastomoses with anterior choroidal artery contribution. ${ }^{12}$ In our series, we mostly found anastomosis between the medial posterior choroidal arteries and the ACA (16 hemispheres, $20 \%$ of cases), as illustrated in Fig $1 B$.

\section{Pio-Pial (Leptomeningeal) Connections between $P C A$ and $A C A$}

In 12 hemispheres (15\%), we also found collaterals between cortical branches of the PCA and cortical branches of the ACA. These are end-to-end anastomoses between cortical branches of the PCA, such as the parieto-occipital artery, and terminal branches of the ACA, which usually develop at the watershed zones, as illustrated in the Fig $1 C$.

\section{Treatment and Clinical Follow-Up}

Among the 56 patients, 23 were treated with antiplatelet therapy. Aspirin was always the first-line treatment. Three of them presented with a recurrent ischemic stroke and were placed under dual antiplatelet therapy. No hemorrhagic stroke was noted under surgical treatment. One patient had a bilateral superficial direct temporal artery-middle cerebral artery anastomosis; 4 others also had a superficial direct temporal artery-MCA bypass, but only at 1 side; and for the last patient, a myo-synangiosis was performed. One patient presented with an ischemic stroke as a complication of the operation, which was contralateral to the operated hemisphere and was imputed to a hypoperfusion during 


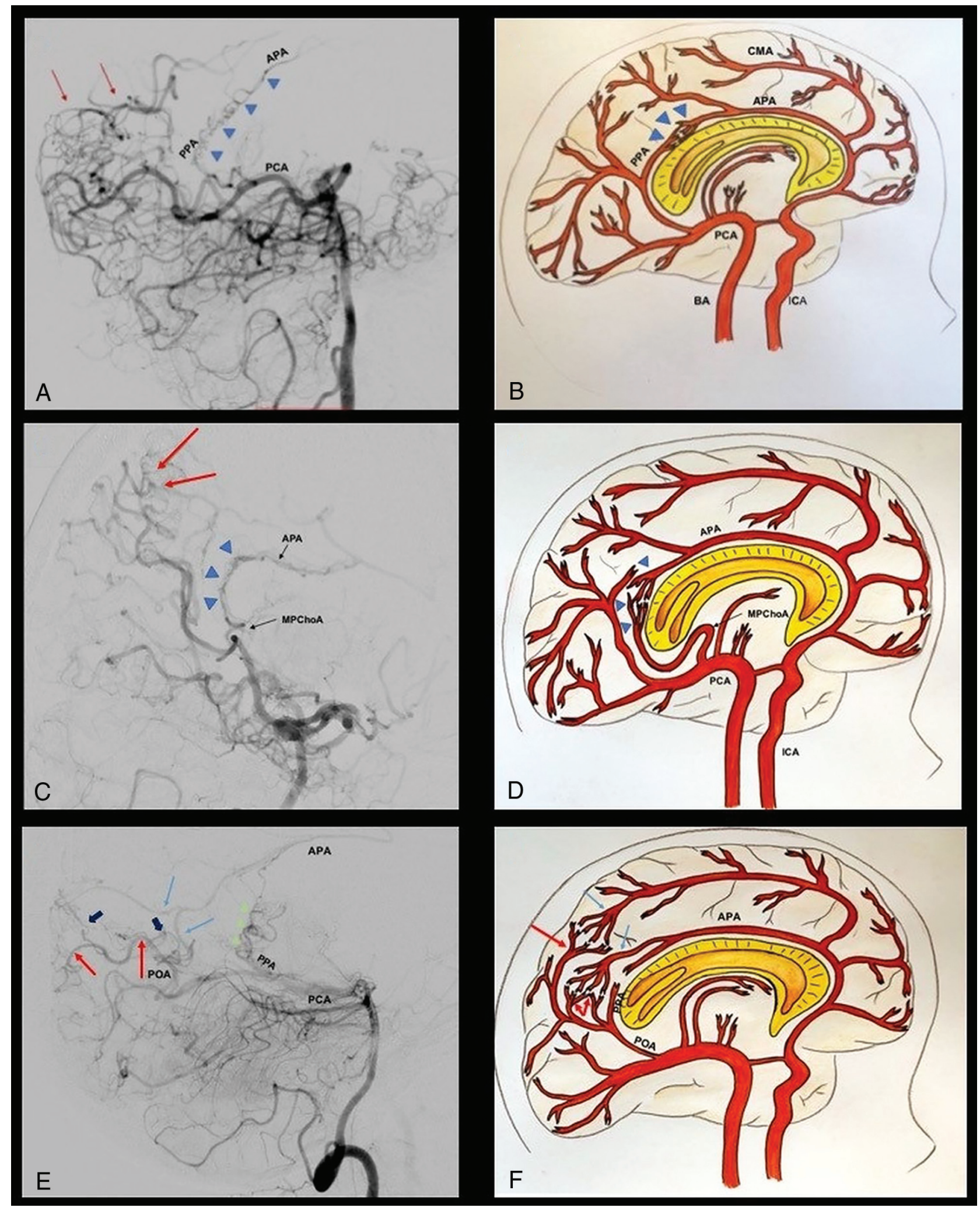

FIG 1. The 3 types of PCA-ACA collaterals are shown by angiograms with corresponding graphic illustrations. The blue triangles ( $A$ and $B)$ indicate the anastomosis between the posterior pericallosal artery and the anterior pericallosal artery (type I). The red arrows indicate the contribution of pio-pial connections to the anastomosis. $C$ and D, Type II collateral, between the medial posterior choroidal artery (MPChoA) and the anterior pericallosal artery (APA) (blue triangles). The MPChoA turns first anteriorly and then backward around the splenium of the corpus callosum to reach the APA. Pio-pial connections are also visible in the angiogram (red arrows). Type III collaterals are visible ( $E$ and $F$ ), where the dark blue arrows indicate pio-pial or leptomeningeal connections between cortical branches from the PCA (red arrows) and cortical branches from the ACA (light blue arrows). The green triangles show the adjunctive presence of posterior pericallosal artery (PPA)-APA connections. POA indicates parieto-occipital artery; CMA, calloso-marginal artery; BA, basilar artery. 


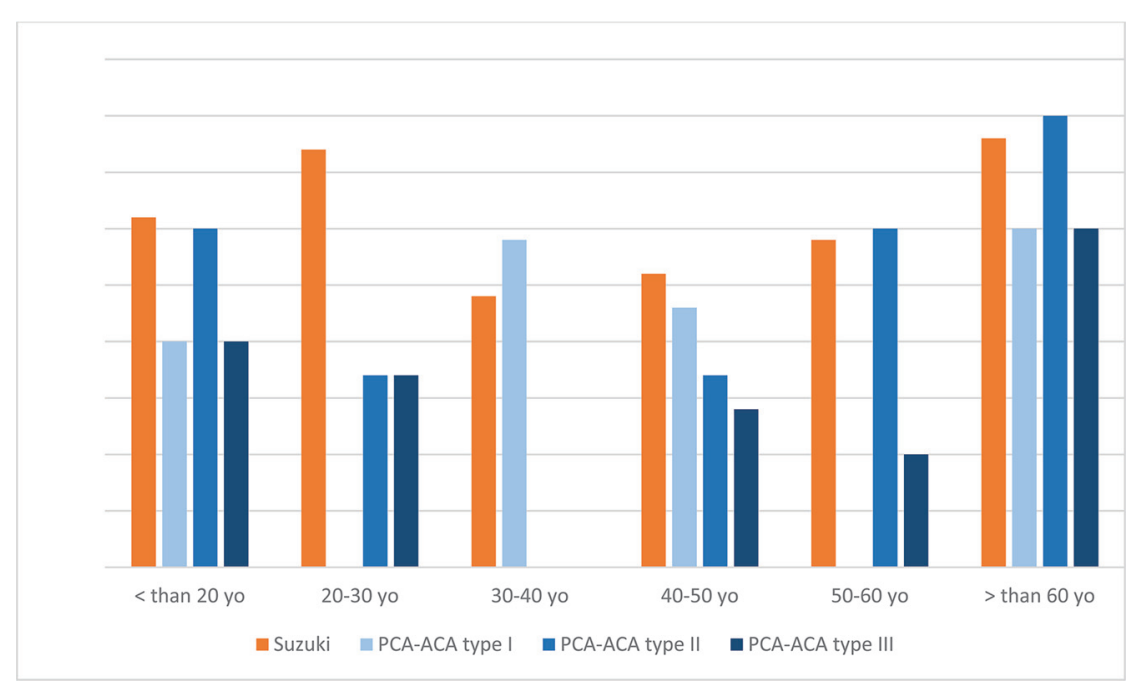

FIG 2. Diagram representation of the distribution of Suzuki scores and PCA-ACA anastomosis depending on the age group.

collaterals that supply the ACA vascular territories, as described by Baltsavias et $\mathrm{al}^{6}{ }^{6}$ a detailed analysis of the involved vessels is not available in the literature.

The first ACA-PCA collaterals found in our series came from branches of the posterior pericallosal artery or splenial artery. This anastomosis between the anterior and posterior circulations is often present in normal hemispheres; ${ }^{13}$ however, it can acquire an important role in Moyamoya disease. In fact, these collaterals could supply, through retrograde flow, the territory of the anterior cerebral artery in case of proximal occlusion of the ICA or if there is poor development of collaterals from the anterior circulation. This posterior pericallosal artery is considered a cortical branch of the parieto-occipital artery, which gives an anastomosis to the anterior pericallosal artery (from the ACA)

the anesthetic induction. The mean follow-up period was 58 months (range, 3-146 months). At the last visit, 14 patients $(25 \%)$ presented with a poor clinical outcome ( $m R S \geq 3$ ). It was directly correlated with the clinical presentation of the patient before the treatment and also with the age at diagnosis of the patient (the older the patient is at diagnosis, the worse the clinical outcome). Among the 14 patients with a poor clinical outcome, the median age was 41 years with only 2 patients younger than 20 years of age.

\section{DISCUSSION}

Moyamoya disease is a progressive neurovascular pathology defined by stenosis of the distal internal carotid artery and middle and anterior cerebral arteries associated with the development of vascular collaterals. ${ }^{1,2,10}$ The progressive stenosis of the internal carotid artery often causes a lack of flow in the vascular territories of the ACA and the MCA., ${ }^{5,7}$ The anastomosis between the posterior and the anterior circulations can provide to refill the territories pertaining to the ACA, especially when the occlusion is proximal to the ophthalmic artery. ${ }^{6,7}$

The scientific literature regarding this pathology is limited because of the rarity of Moyamoya disease, ${ }^{4}$ and most articles are dedicated to the description of the surgical techniques and their outcomes. Only a few authors ${ }^{6-9}$ have provided detailed angiographic descriptions of the different types of collaterals that naturally develop. Thus, many questions related to the frequency, presence of repeatable patterns, and associations of collaterals with specific phases of the pathology still remain open.

A great contribution to our knowledge was by Baltsavias et $\mathrm{al},{ }^{6,7}$ who classified the different anastomoses into 2 superficialmeningeal systems (leptomeningeal or pio-pial and duro-pial) and 2 deep parenchymal systems (subependymal and thalamic). Among these systems, we decided to analyze the one between the posterior cerebral artery and the anterior cerebral artery. Although it is known that the posterior circulation may provide dorsal to the splenium of the corpus callosum. ${ }^{12}$ Zeal and Rhoton ${ }^{14}$ showed that this posterior pericallosal artery could also arise from the calcarine (12\%), choroidal (12\%), or posterior temporal (6\%) branches of the PCA. This direct anastomosis between anterior and posterior pericallosal arteries contributes to the pial network around the splenium of the corpus callosum, which could be interpreted as one of the remnants of the embryonic limbic arch. ${ }^{15}$

The other frequent ACA-PCA anastomosis we found in our series is the connection between the posterior choroidal arteries and the anterior pericallosal artery. The posterior choroidal arteries, first described by Abbie in $1934,{ }^{16}$ are usually connected to the anterior circulation within the choroid plexus. In fact, the latter is usually supplied anteriorly by the anterior choroidal artery and the ACA and posteriorly by the posterior choroidal arteries. Our hypothesis to explain the development of these collaterals comes from the analysis of the embryologic stage of arterial development. In the early choroidal stage, the choroidal plexus is principally supplied by choroidal branches of the anterior cerebral and anterior choroidal arteries that anastomose near the interventricular foramen. ${ }^{17}$ Then, the posterior choroidal arteries develop from the caudal division of the internal carotid artery (future posterior cerebral artery) and also supply the choroidal plexus of the lateral and third ventricles, creating other anastomoses to branches of the ACA and anterior choroidal artery. At this telencephalic stage (before the complete development and closure of the corpus callosum), the choroidal plexus of the lateral and third ventricles is consequently supplied by choroidal branches from 3 different systems (the anterior cerebral, anterior choroidal, and posterior choroidal arteries) and the interventricular foramen is an important place of anastomosis between these branches. During the progressive closure of the corpus callosum, choroidal branches of the ACA are elongated at the dorsal aspect of the splenium, and this elongation contributes to the regression of these choroidal branches. 


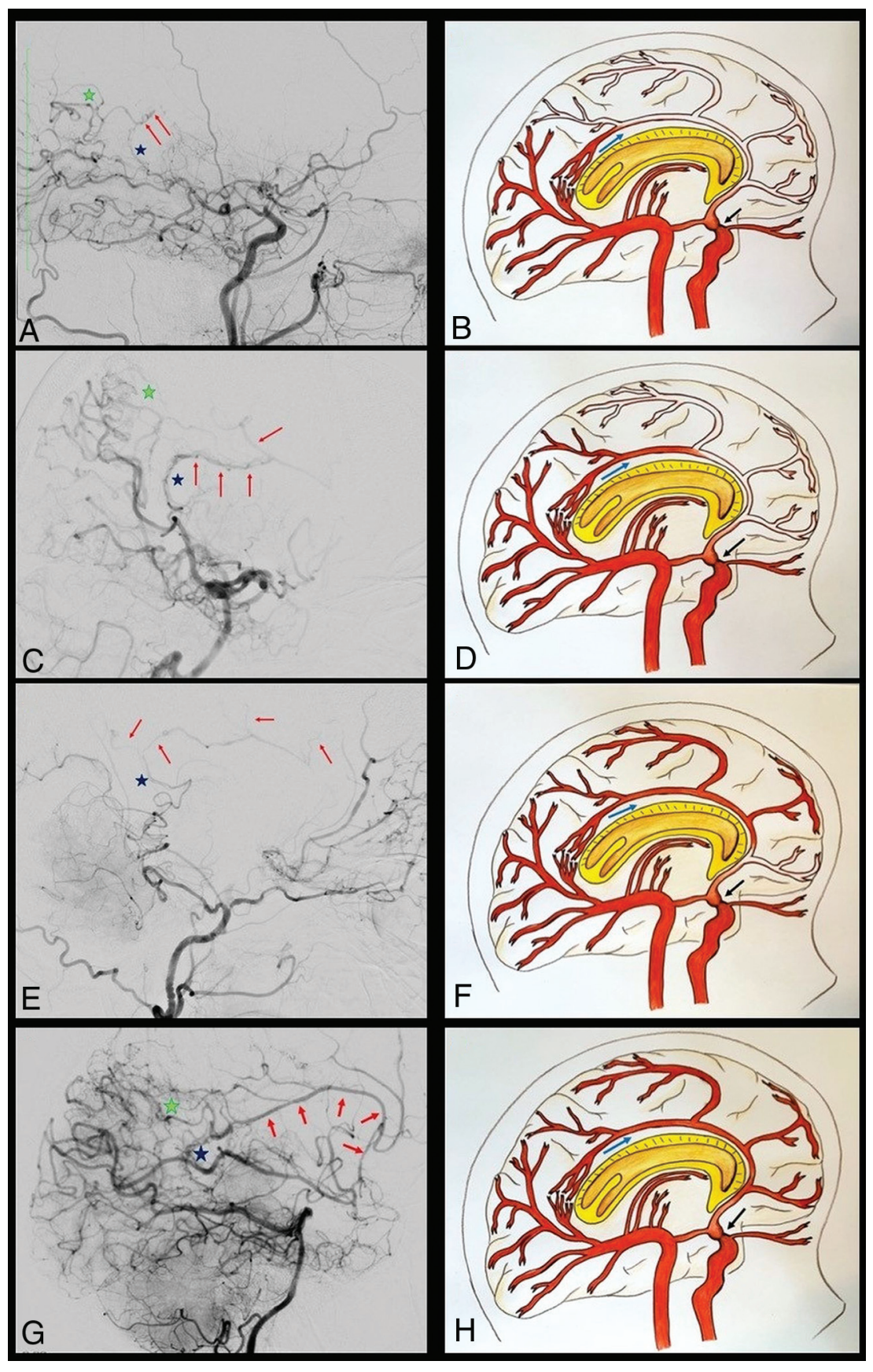

FIG 3. $A-D$, The angiograms and graphic illustrations show the capacity to compensate the ACA territories by the PCA-ACA anastomoses in case of proximal ICA stenosis (black arrow), through a 4-grade classification. In grade I ( $A$ and $B$ ), the collaterals refill just the first part of the ACA, without seeing any cortical branch. The angiogram on the left shows that the contribution to the refilling is made by the posterior pericallosal artery (blue star) and by pio-pial connections (green star). $C$ and $D$, The retrograde flow reaches a larger part of the ACA, also highlighting a cortical branch of the ACA (grade II). A double contribution from the pio-pial connection (green star) and medial posterior choroidal artery (blue star) is visible in the angiogram. Grade III (E and F) consists of the retrograde refilling of 2 or 3 ACA branches (red arrows in E), which, in this case, are supported by a medial posterior choroidal artery-anterior pericallosal artery anastomosis. In grade IV, almost all the ACA territory is retrogradely refilled ( $G$ and $H$ ). The green and blue stars indicate the 2 main connections that compensate the ACA territories hypoperfusion.

Simultaneously, the choroidal territory of the ACA branches is competitively supplied by branches of the posterior choroidal arteries.
The fact that the choroidal plexus is initially served mainly by branches of the ACA and that these are progressively replaced by branches of the PCA explains the possibility of finding this type of ACA-PCA anastomosis in the adult configuration. The anastomotic system between these 2 arteries is under the control of an embryonic arterial arcade or its remnants, known as the "limbic arterial arch." This arch was first described by Moffat ${ }^{18}$ in 1961 and usually represents a transient stage during ontogenesis; however, sometimes it can also persist in adults. Lasjaunias et $\mathrm{al}^{12}$ described 2 types of persisting limbic arch: the first or "true" limbic arch links the anterior choroidal artery to the ACA around the limbic structures; in the second one, the PCA takes over the anterior choroidal artery role and is linked to the ACA. In this latter case, the arch supplies the corpus callosum and multiple connections are present between the ACA and posterior choroidal artery passing around the splenium of the corpus callosum. Our impression is that the choroidal collaterals we found are linked to the ACA through the limbic arterial arch (type II). This arch could persist as described by Lasjaunias et $\mathrm{al}^{12}$ or could be restored after a chronic ischemic stimulus.

Another type of collateral described in our series is a pio-pial or leptomeningeal connection between the parenchymal branches of the posterior cerebral artery and posterior branches of the anterior cerebral artery. This kind of anastomosis has already been described by Baltsavias et $\mathrm{al}^{6,7}$ and mostly consists of the anastomosis between PCA and ACA parietal branches or between PCA and MCA parietal or temporal branches at the watershed zones.

Another possible anastomosis between the ACA and PCA is a choroidal anastomosis located at the level of the interventricular foramen. No case presenting this anastomosis was found in our series, probably only due to the absence of selective DSA. This is a possible anastomosis between the posterior choroidal arteries and hypothalamic branches of the anterior communicating artery. This anastomosis could also be explained by the embryologic vascularization of the choroidal plexus and the remnant of the fornix-choroidal 
perforators. ${ }^{16,17}$ Such branches and anastomosis have been described in other pathologies such as choroidal arteriovenous malformations or vein of Galen malformations. ${ }^{12}$

Our hypothesis is that after an ischemic stimulus from these regions, the vascular system responds by using a pre-existing anastomosis, whose flow could be improved by neoangiogenesis, to refill retrogradely the ACA territories.

We also observed that most of these collaterals from the posterior circulation were present in advanced stages of the disease. It may suggest the necessity of supplying the ACA territories when the ICA stenosis becomes more severe and does not allow the ophthalmic artery to sufficiently supply the ACA territories through its collaterals.

The analysis of our results shows that the anastomotic circles were present in $10 \%$ of cases in Suzuki stage IV ( 8 hemispheres) and in $10 \%$ in Suzuki ${ }^{13}$ stage VI ( 8 hemispheres). These connections were less common in stage 0 ( 1 hemisphere, $1.25 \%$ ), stage I (1 hemisphere, 1.25\%), stage II (4 hemispheres, $5 \%$ ), stage III (4 hemispheres, $5 \%$ ) and stage $\mathrm{V}$ (4 hemispheres, $5 \%$ ).

Our impression is that the lack of blood supply in the ischemic areas stimulates the development of collaterals in patients affected by Moyamoya disease. The aim of this study was to give a more detailed view of the possible anastomoses between the posterior and the anterior cerebral circulations, to understand the evolution of these collaterals to better manage these patients.

\section{Limitations}

Our study is retrospective, based on conventional cerebral DSA, without selective injection to evaluate the anastomosis between the posterior cerebral artery and the anterior cerebral artery. The other important limitation is that most of our patients had only 1 conventional diagnostic DSA and thereafter were followed by MR imaging. Consequently, it is not possible to evaluate the development of the collateral circles during the evolution of Moyamoya disease.

\section{CONCLUSIONS}

We found 3 different types of anastomoses between the posterior and the anterior circulations, with different abilities to compensate retrogradely the anterior circulation. These collaterals are frequent in Moyamoya disease, particularly in advanced Suzuki stages IV to VI. Their development depends on the lack of blood supply to the ACA territories and on the ability to compensate the other 3 collateral systems (ophthalmic-ACA anastomosis, duro-pial anastomosis, and leptomeningeal anastomosis). The analysis of these collateral circles through selective contrast injection into the posterior cerebral artery and detailed analysis of the other anastomotic circles would be helpful to understand the natural history of the disease and to better select patients who need bypass surgery.

Disclosures: Alexander G. Weil-UNRELATED: Employment: Dr. A. G. Weil, Inc Michael Reinert-UNRELATED: Grants/Grants Pending: Neuro-oncology. *Michel Bojanowski-UNRELATED: Employment: Center Hospitalier de l'Université de Montréal. *Money paid to the Institution.

\section{REFERENCES}

1. Bang OY, Fujimura M, Kim SK. The pathophysiology of Moyamoya disease: an update. J Stroke 2016;18:12-20 CrossRef Medline

2. Burke GM, Burke AM, Sherma AK. Moyamoya disease: a summary. Neurosurg Focus 2009;26:E11 CrossRef Medline

3. Scott RM, Smith ER. Moyamoya disease and moyamoya syndrome. N Engl J Med 2009;360:1226-37 CrossRef Medline

4. Kim JS. Moyamoya disease: epidemiology, clinical features, and diagnosis. J Stroke 2016;18:2-11 CrossRef Medline

5. Kwag HJ, Jeong DW, Lee SH, et al. Intracranial hemodynamic changes during adult moyamoya disease progression. J Clin Neurol 2008;4:67-74 CrossRef Medline

6. Baltsavias G, Khan N, Filipce V, et al. Selective and superselective angiography of pediatric moyamoya disease angioarchitecture in the posterior circulation. Interv Neuroradiol 2014;20:403-12 CrossRef Medline

7. Baltsavias G, Khan N, Valavanis A. The collateral circulation in pediatric moyamoya disease. Childs Nerv Syst 2015;31:389-98 CrossRef Medline

8. Robert T, Ciccio G, Sylvestre P, et al. Anatomic and angiographic analyses of ophthalmic artery collaterals in Moyamoya disease. AJNR Am J Neuroradiol 2018;39:1121-26 CrossRef Medline

9. Matsushima Y, Inaba Y. The specificity of the collaterals to the brain through the study and surgical treatment of moyamoya disease. Stroke 1986;17:117-22 CrossRef Medline

10. Suzuki J, Takaku A. Cerebrovascular "moyamoya" disease: disease showing abnormal net-like vessels in base of brain. Arch Neurol 1969;20:288-99 CrossRef Medline

11. Rhoton AL Jr. The supratentorial arteries. Neurosurgery 2002;51:S153-20 CrossRef Medline

12. Lasjaunias P, Brugge KGt, Berenstein A. Surgical Neuroangiography. New York: Springer-Verlag; 2006

13. Kahilogullari G, Comert A, Ozdemir M, et al. Arterial vascularization patterns of the splenium: an anatomical study. Clin Anat 2013;26:675-81 CrossRef Medline

14. Zeal AA, Rhoton AL Jr. Microsurgical anatomy of the posterior cerebral artery. J Neurosurg 1978;48:534-59 CrossRef Medline

15. Ture U, Yasargil MG, Krisht AF. The arteries of the corpus callosum: a microsurgical anatomic study. Neurosurgery 1996;39:1075-84; discussion 1084-85 CrossRef Medline

16. Abbie AA. The morphology of the fore-brain arteries, with especial reference to the evolution of the basal ganglia. J Anat 1934;68:43370 Medline

17. Padget DH. The development of the cranial arteries in the human embryo. Contributions to Embryology 1948;32:205-06

18. Moffat DB. The development of the posterior cerebral artery. $J$ Anat 1961;95:485-94 Medline 